\title{
EXPECTED VALUES AND VARIANCES IN BOOKMAKER PAYOUTS: A THEORETICAL APPROACH TOWARDS SETTING LIMITS ON ODDS
}

\author{
Dominic Cortis* \\ University of Leicester, University of Malta
}

\begin{abstract}
This study summarizes the key methods of displaying probabilities as odds and provides simple mathematical derivation of a number of key statements in setting odds. Firstly it estimates the expected bookmaker profit as a function of wagers placed and bookmaker margin. Moreover it shows that odds set by bookmakers should have implied probabilities that add up to at least one, otherwise arbitrage is present. Bookmakers can increase profitability by offering more multiples, also known as accumulators, and can lower variation in payouts by maintaining the ratio of wagers and implied probability per outcome.
\end{abstract}

Key Words: betting markets, odds, arbitrage, multiples, accumulators, bookmakers

\section{INTRODUCTION}

The speculative nature of betting markets has led to significant research ranging from their relationship with external forces (Braun \& Kvasnicka, 2013) to insider trading (Schnyzter \& Shilony, 1995; Shin, 1993; Vaughan Williams \& Paton, 1997). Specifically, a great deal of research has focused on the market efficiency of betting markets (Brown \& Abraham, 2002; Cortis, Hales, \& Bezzina, 2013; Deschamps \& Gergaud, 2007; Easton \& Uylangco, 2007; Gandar, Zuber, \& Johnson, 2004; Gil \& Levitt, 2007; Hvattum, 2013; Paul \& Weinbach, 2002; Terrell \& Farmer, 1996; Woodland \& Woodland, 2001). While techniques used within these market efficiency inquiries differ greatly, there is one key common question that is being asked: "Do odds on different outcomes represent the actual probabilities correctly?".

A crucial challenge to any reader in betting related research is the lack of consistency in the notation and the formalisation of the key concepts.

\footnotetext{
* Corresponding author: Dominic Cortis, Room 104, College House, Department of Mathematics, University of Leicester, University Road, UK LE1 7RH

Email: dc156@le.ac.uk
} 
Furthermore any derivations of expected profit tend to focus on discrete bestestimate values rather than providing a range of results. This paper attempts to address this by firstly providing an overview of how different types of odds are determined by their respective probabilities. This acts as introduction to readers who are new to the area and leads to the derivations of the restrictions on and profitability of a bookmaker when setting odds, including the occurrence of arbitrage. ${ }^{1}$ These two sections can be considered as an extension Levitt's (2004) work on bookmakers' profitability when pricing events with binary outcomes to events with more than two.

Subsequently these results are extended to multiples, also referred to as accumulators, another type of bet that has become significantly popular. Zafiris (2014) gave special attention to this type of bet by showing the net expected profit from a bettor's perspective. This research paper evaluates the payout from a bookmaker's standpoint and extends it to a more general form. The findings presented here are mainly theoretical and include mathematical proofs but they are also backed up by simple examples throughout the script.

\section{EVALUATING ODDS}

The odds on an event represent the probability of different outcomes and are used instead percentages as they display the return on a wager. There are three main styles of displaying odds: European, English or American odds and three other styles that are mainly popular in Asia: Hong Kong, Indonesian and Malaysian odds. The majority of online betting sites offer the facility for clients to display odds according to their preferred format between the three main styles. Throughout this report, any reference to odds will be to European odds unless otherwise stated.

American odds, also known as money line odds, can be shown as positive or negative figures. A positive figure shows how much would be won on a wager of 100 units while a negative odd shows how much needs to be wagered in order to win 100 units. Positive figures are generally used for outcomes that are less than $50 \%$ likely to occur while negative figures are generally used for outcomes that are more than 50\% likely to occur. English or fractional odds show the amount that would be won excluding the wager as a ratio to the wager with both values being integers. They can be calculated as the ratio of the probability of an outcome not occurring to the probability of an outcome occurring. European odds are also called decimal odds and represent the odd of an event occurring as the inverse of its probability.

Hong Kong odds show the winnings, net of the wager, made on a wager of one unit. Therefore it is always one less than the European odd. Indonesian odds are similar to American odds but relate to the amount won or to be wagered to win one unit. Finally Malaysian odds show the winnings, net of

\footnotetext{
1 Arbitrage is defined as being able to make a series of bets that would result in riskless profit for a bettor.
} 
the wager, made on a wager of one in the case of likely outcomes and the amount to be wagered in order to win one unit, excluding own wager, in case of unlikely outcomes. In essence Hong Kong odds are equal to Indonesian and Malaysian odds for unlikely and likely outcomes respectively.

Mathematically the odd on outcome $i$ with probability $\pi_{i}$ to occur would be shown as below:

American / Money Line $\left\{\begin{array}{l}+100 \frac{1-\pi_{i}}{\pi_{i}} \text { for } \pi_{i} \leq 0.5 \\ \text { Odd: }\end{array} \quad\right.$ for $\pi_{i}>0.5$

English / Fractional Odd: $\quad\left(1-\pi_{i}\right) / \pi_{i}$

European/Decimal Odd: $\frac{1}{\pi_{i}}$

Hong Kong Odd:

$$
\frac{1-\pi_{i}}{\pi_{i}}=\frac{1}{\pi_{i}}-1
$$

Indonesian Odd:

$$
\begin{cases}+\frac{1-\pi_{i}}{\pi_{i}} & \text { for } \pi_{i} \leq 0.5 \\ -\frac{\pi_{i}}{1-\pi_{i}} & \text { for } \pi_{i}>0.5\end{cases}
$$

$$
\begin{cases}-\frac{\pi_{i}}{1-\pi_{i}} & \text { for } \pi_{i} \leq 0.5 \\ +\frac{1-\pi_{i}}{\pi_{i}} & \text { for } \pi_{i}>0.5\end{cases}
$$

For example consider an outcome that has a $20 \%$ chance of occurring. The American, English, European, Hong Kong, Indonesian and Malaysian odds be displayed as $100\left(\frac{1-0.2}{0.2}\right)=+400,(1-0.2) / 0.4=4 / 1, \frac{1}{0.2}=5, \frac{0.8}{0.2}=4$, +4 and $-\frac{0.2}{0.8}=-0.25$ respectively. This can be interpreted as a successful one unit wager on this outcome resulting in receiving five units back (European/Decimal), consisting of the own stake and a winning of four units (English/Fractional, Hong Kong, Indonesian) resulting in a net stake of 25\% of winnings (Malaysian) or a $400 \%$ profit on the stake (American/Money Line). A negative money line such as -300 can be interpreted as needing to 
stake 300 units in order to win one hundred units. Similarly this would be -3 in an Indonesian odd.

\section{BOOKMAKER MARGIN}

The probabilities of all possible mutually exclusive outcomes within a probability space of an event add up to $100 \%$. However bookmakers do not operate in a 100\% scenario (Archontakis \& Osborne, 2007; Cortis, Hales, \& Bezzina, 2013; Štrumbelj, 2014).

For example consider a probability space for an event with two equal outcomes. Under a fair market, the (European) odds would be equal to 2 for each outcome. However a bookmaker would price the odd a bit lower, say 1.9. This means that having received two wagers of one unit on each outcome, the bookmaker would pay out 1.9 units and make a profit of 0.1 units.

The inverse of European odds, being the implied probabilities, adds up to more than $100 \%$, and the difference to $100 \%$ is generally described as the bookmaker margin (Cain, Law, \& Peel, 2003; Peel \& Thomas, 1992; Štrumbelj, 2014). Other similar terms include the over-round (Zafiris, 2014) and the vig (Peel \& Thomas, 1992).

A real-life example can be seen in Figure 1. Using the example of Manchester City - Liverpool, the state space consists of a home team (Manchester City) win, draw or away team win. The sum of the inverse of probabilities are equal to $\frac{1}{1.86}+\frac{1}{3.75}+\frac{1}{4.05}=1.05121 \%$. The bookmaker margin in this case is $5.121 \%$.

Figure 1: European Odds for Two Matches as shown on Betsson.com as at 16:00 on 25th August 2014

\begin{tabular}{ll|l|l|l|l|}
\hline $19: 00$ & Real Madrid - Córdoba & 1.05 & 14.50 & 30.00 & liue \\
\hline $20: 00$ & Manchester City - Liverpool & 1.86 & 3.75 & 4.05 & liue \\
\hline
\end{tabular}

The fact that implied probabilities from a bookmaker do not necessary add up to one leads to two key concerns: is it necessary for implied probabilities to add up to more than $100 \%$ and how does this difference between real and implied probabilities affect bookmaker profitability?

\section{OUTCOMES ON A SINGLE EVENT}

As an initial examination, this section deals with setting odds for one event. Prior to examining the bookmaker's profit, the restrictions on setting odds are proved.

ASSUMPTION 1: All odds are provided by one bookmaker. 


\section{EXPECTED VALUES AND VARIANCES IN BOOKMAKER PAYOUTS}

ASSUMPTION 2: All probabilities, implied or actual, relate to one event with a probability space of $n$ mutually exclusive outcomes.

\subsection{RESTRICTIONS ON SETTING ODDS}

DEFINITION 1: $\quad$ Let $\pi_{i}$ represent the implied probability, calculated as the inverse of the European Odd, of outcome $i$ occurring where $i \in\{1, \ldots, n\}$.

DEFINITION 2: $\quad$ Let $p_{i}$ represent the actual known probability of outcome $i$ occurring where $i \in\{1, \ldots, n\}$.

DEFINITION 3: Let $w_{i}$ be the total value in units of wagers placed on outcome $i$.

ASSUMPTION 3: The bookmaker is aware of the real value of $p_{i}$ and can control $\pi_{i}$ but not $w_{i} \forall i \in\{1, \ldots, n\}$.

DEFINITION 4: $\quad$ Let $P$ be the random variable representing the profit made by the bookmaker for bets made on one event.

$$
\therefore \mathrm{E}(P)=E(\text { Wagers })-E(\text { Payouts })=\sum_{i=1}^{n} w_{i}-\sum_{i=1}^{n} \frac{w_{i} p_{i}}{\pi_{i}}
$$

PROPOSITION 1. If the implied odds of an event add up to less than one, then arbitrage exists.

Proof (By contradiction): We need to find a strategy for a bettor that would result in a certain loss for the company $[P<0]$. We know that $\sum_{i=1}^{n} \pi_{i}<1$. Assume a strategy such that $w_{i}=\pi_{i}$. Therefore for any outcome the payout by the company to the bettor is $w_{i} \times \frac{1}{\pi_{i}}=1$.

$$
\sum_{i=1}^{n} \pi_{i}<1 \Rightarrow P=\text { Wagers }- \text { Payouts }=\sum_{i=1}^{n} w_{i}-1=\sum_{i=1}^{n} \pi_{i}-1<0
$$

PROPOSITION 2. A bookmaker would risk a negative expected profit if the implied probability of any outcome is lower than the actual probability.

Proof (By contradiction): Let $p_{q}>\pi_{q}$ for some $q \in\{1, \ldots, n\}$. Assume a single wager of 1 on outcome q.

$$
\mathrm{E}(P)=E(\text { Wagers })-E(\text { Payouts })=1-\frac{p_{q}}{\pi_{q}}<0
$$

Each of these propositions lead to two important restrictions for bookmakers when setting odds for each outcome of an event: these must add 
up to at least one and should not be smaller than the actual probability. Therefore the relationship shown in equation 8 can be set.

$$
\pi_{i}=p_{i}\left(1+k_{i}\right) \text { where } k_{i}>0 \& i \in\{1, \ldots, n\} .
$$

\subsection{BOOKMAKER PROFIT AND DEVIATION}

It is very common for $k$ to be treated as a constant, that is maintaining the same ratio of implied to actual probability for all outcomes (e.g. Archontakis \& Osborne, 2007; Cortis, Hales, \& Bezzina, 2013; Goddard \& Asimakopoulos, 2004; Zafiris, 2014). Contrastingly Shin (1993) demonstrates how bookmakers can evaluate odds in order to minimize the effects of insider training and Štrumbelj (2014) further proves that using Shin's technique on implied odds resulted in a better forecasting accuracy of real probabilities of fixed odds over a series of 48,126 matches in five sports.

Koch \& Shing (2008) explain that bookmakers are limited to a finite list of odds, especially if they operate in an English/Fractional odd environment since odds are either rounded up or possibly limited by regulation. They report finding 88 odd levels in UK horse racing betting markets between 2001 and 2006. A similar limitation, albeit to a lower extent, can be found for European/Decimal odds. For example it will be impossible for an implied probability to be $75 \%$ since this would result in a recurring decimal odd (1. $\dot{3}$ ). For example, an examination of 9,120 European odds provided by eight bookmakers $^{2}$ for the starting odds of the 380 matches $^{3}$ of all 2013/14 English Premiership results in only 588 unique odds. This may mean that bookmakers, given a particular bookmaker margin, are likely to round actual probabilities to implied probabilities to the nearest value in a grid or use particular set doubles $^{4}$ and triples of odds, rather than adjusting solely for insider trading.

The bookmaker's profit is dependent on the amount and spread of wagers, which are dependent on consumer preference, and the bookmaker margin, which is set internally. Although bookmakers face challenges in the deviation of possible profits, this has been generally disregarded in academic work. This section initially evaluates the distribution of profit in the case of a bookmaker margin that is variable for different outcomes and then simplifies it to a constant bookmaker margin applied to all outcomes.

\footnotetext{
${ }^{2}$ Bet365, Bwin, Interwetten, Ladbrokes, Pinnacle Sports, Stan James, VC Bet, and William Hill.

${ }^{3}$ Each match has three possible outcomes: home team win, away team win or draw. Odds ranged from 1.1 to 29 .

${ }^{4}$ Consider an event with two mutually-exclusive outcomes and a bookmaker margin of around 5\%. Some likely doubles, representing the European/Decimal odds for the two outcomes, could be $1.8-2,1.6-2.25$ and $1.55-2.5$.
} 
PROPOSITION 3. If $\pi_{i}=p_{i}\left(1+k_{i}\right)$ where $k_{i}>0 \& i \in\{1, \ldots, n\}$ then $P \sim N\left(\sum_{i=1}^{n} \frac{k_{i} w_{i}}{1+k_{i}}, \sum_{i=1}^{n} w_{i} \sum_{i=1}^{n} \frac{w_{i}\left(k_{i}-1\right)}{1+k_{i}}+\sum_{i=1}^{n} \frac{w_{i}^{2}}{\left(1+k_{i}\right) \pi_{i}}-\left(\sum_{i=1}^{n} \frac{k_{i} w_{i}}{1+k_{i}}\right)^{2}\right)$

Proof: $E(P)=E($ Wagers $)-E($ Payouts $)=\sum_{i=1}^{n} w_{i}-\sum_{i=1}^{n} \frac{w_{i} p_{i}}{\pi_{i}}$

$$
E(P)=\sum_{i=1}^{n} w_{i}-\sum_{i=1}^{n} \frac{w_{i} p_{i}}{p_{i}\left(1+k_{i}\right)}=\sum_{i=1}^{n} \frac{k_{i} w_{i}}{1+k_{i}}
$$

$E\left(P^{2}\right)=E\left(\right.$ Wagers $\left.^{2}\right)-2 E($ Wagers $) E($ Payouts $)-E\left(\right.$ Payout $\left.^{2}\right)$

$E\left(P^{2}\right)=\left(\sum_{i=1}^{n} w_{i}\right)^{2}-2 \sum_{i=1}^{n} w_{i} \sum_{i=1}^{n} \frac{w_{i} p_{i}}{\pi_{i}}+\sum_{i=1}^{n} \frac{w_{i}^{2} p_{i}}{\pi_{i}^{2}}$

$E\left(P^{2}\right)=\left(\sum_{i=1}^{n} w_{i}\right)^{2}-2 \sum_{i=1}^{n} w_{i} \sum_{i=1}^{n} \frac{w_{i}}{1+k_{i}}+\sum_{i=1}^{n} \frac{w_{i}^{2} p_{i}}{\left(1+k_{i}\right)^{2} p_{i}^{2}}$

$E\left(P^{2}\right)=\left(\sum_{i=1}^{n} w_{i}\right)^{2}-2 \sum_{i=1}^{n} w_{i} \sum_{i=1}^{n} \frac{w_{i}}{1+k_{i}}+\sum_{i=1}^{n} \frac{w_{i}^{2}}{\left(1+k_{i}\right) \pi_{i}}$

$E\left(P^{2}\right)=\sum_{i=1}^{n} w_{i} \sum_{i=1}^{n} \frac{w_{i}\left(k_{i}-1\right)}{1+k_{i}}+\sum_{i=1}^{n} \frac{w_{i}^{2}}{\left(1+k_{i}\right) \pi_{i}}$

$\operatorname{Var}(P)=E\left(P^{2}\right)-[E(P)]^{2} \mathrm{VV}$

$\operatorname{Var}(P)=\sum_{i=1}^{n} w_{i} \sum_{i=1}^{n} \frac{w_{i}\left(k_{i}-1\right)}{1+k_{i}}+\sum_{i=1}^{n} \frac{w_{i}^{2}}{\left(1+k_{i}\right) \pi_{i}}-\left(\sum_{i=1}^{n} \frac{k_{i} w_{i}}{1+k_{i}}\right)^{2}$

PROPOSITION 4. If the implied probability of any outcome is lower or equal to than the actual probability, then the bookmaker is expected to have a profit.

Proof: $\quad p_{q} \leq \pi_{q} \Rightarrow \quad \pi_{i}=p_{i}\left(1+k_{i}\right) \quad$ for $\quad k_{i}>0 \quad \Rightarrow E(P)=$ $\sum_{i=1}^{n} \frac{k_{i} w_{i}}{1+k_{i}} \geq 0$.

Proposition 4 leads to an extension over Proposition 2 since it shows that expected bookmaker profits are positive if implied probabilities are larger than actual probabilities. A special case, discussed earlier, is when $k$ is constant. In this case Proposition 3 can easily be extended as shown in equation 9 (a proof from first principles is given in Appendix A).

$$
P \sim N\left(\frac{k}{1+k} \sum_{i=1}^{n} w_{i}, \sum_{i=1}^{n} \frac{w_{i}^{2}}{(1+k) \pi_{i}}-\left(\frac{\sum_{i=1}^{n} w_{i}}{1+k}\right)^{2}\right)
$$

The evaluation of the distribution for a constant bookmaker margin leads to three observations. Firstly Proposition 4 shows a relaxation to Assumption 3 in that the bookmaker does not need to know the exact value of the actual probability for each outcome. 
Another important outcome is that the percentage expected profit for a betting company is $\frac{k}{1+k}$. For example, considering again an event that can result in one of two equally likely outcomes but is sold at an odd of 1.8, the bookmaker margin $k=\frac{1}{1.8}+\frac{1}{1.8}-1=\frac{10}{9}-1=\frac{1}{9}$. We would expect equal amounts to be wagered on each outcome since they are equally likely. Assuming a wager of one unit on each outcome, the wagers add up to two while the payout is 1.8 . This is a profit of 0.2 from two units which is $10 \%$. This is equivalent to $\frac{k}{1+k}=\frac{\frac{1}{9}}{\frac{10}{9}}=\frac{1}{10}$.

In the example shown in Figure 1, the bookmaker margin was 5.121\% and therefore the expected profit is $4.872 \%$. This simple calculation is important to point out as at times the bookmaker margin may be confused with expected profits.

PROPOSITION 5 Certain profit is made if wagers on each outcome are distributed in proportion to the probabilities of each outcome.

Proof: $\quad$ Assume $k>0, \sum_{i=1}^{n} w_{i}>0, w_{i}=b_{i} \sum_{i=1}^{n} w_{i}$ and $\sum_{i=1}^{n} b_{i}=1$.

The risk is minimised if there are no fluctuations, therefore if there is no variance.

$\operatorname{Var}(P)=0$

$$
\begin{gathered}
\frac{1}{(1+k)^{2}}\left(\sum_{i=1}^{n} \frac{w_{i}^{2}}{p_{i}}-\left(\sum_{i=1}^{n} w_{i}\right)^{2}\right)=0 \\
\Rightarrow\left(\sum_{i=1}^{n} \frac{\left(b_{i} \sum_{i=1}^{n} w_{i}\right)^{2}}{p_{i}}-\left(\sum_{i=1}^{n} w_{i}\right)^{2}\right)=0 \\
\left(\sum_{i=1}^{n} w_{i}\right)^{2}\left(\sum_{i=1}^{n} \frac{b_{i}^{2}}{p_{i}}-1\right)=0 \\
\Rightarrow\left(\sum_{i=1}^{n} \frac{b_{i}^{2}}{p_{i}}-\sum_{i=1}^{n} p_{i}\right)=0 \\
\sum_{i=1}^{n} \frac{b_{i}^{2}-p_{i}^{2}}{p_{i}}=0
\end{gathered}
$$

One of the solutions for the above is $b_{i}=p_{i}$ where $k_{i}>0 \& i \in$ $\{1, \ldots, n\}$.

Proposition 5 is equivalent to Levitt's (2004) first scenario in which bookmakers sustain profits by maintaining the proportion of money bet on each side of an equally likely binary outcome. Consider that in the Manchester City - Liverpool example, if a total of 51,25 and 23 units are wagered on the home team winning, a draw or the away team winning 
respectively; the company would have received 99 units in total wagers but would pay a maximum of 94.86 units, resulting in certain profit. $^{5}$

Bookmakers may be tempted to adjust odds according to the volume of wagers made on different outcomes. One can point out that there are two rationales for this. The general public may have better information and are therefore better at estimating actual probabilities. Secondly the bookmaker may wish to diminish the likelihood of create arbitrage when its odds are compared to others. However this proposition provides a third important justification for bookmakers to adjust odds according to the volume of wagers made.

\section{MULTIPLES}

A Multiple is a bet with a pay-out out contingent on two or more independent outcomes. For example, as shown in Figure 1, a bettor can place a multiple wager on Real Madrid and Manchester City winning their respective matches for an odd of $1.05 \times 1.86=1.953$. This bet is paid off only if both Real Madrid and Manchester City win their respective matches.

DEFINITION 5: Let $P^{(m)}$ be the random variable representing the profit made by the bookmaker on bets over a multiple with $m$ events.

In essence a multiple of $m$ events with $n_{m}$ outcomes per event, can be represented as one outcome with $\prod_{j=1}^{m} n_{m}$ outcomes and a margin of $(1+$ $\left.k_{m}\right)^{m}-1$ where $k_{m}$ is the geometric mean of the bookmaker margin for each event. This means that it is easy to prove that

$$
P^{(m)} \sim N\left(\frac{G-1}{G} \sum W, \frac{1}{G} \sum \frac{W^{2}}{\pi^{(m)}}-\left(\frac{\sum W}{G}\right)^{2}\right)
$$

where

$$
\begin{array}{r}
\sum W=\sum_{i_{1}=1}^{n_{1}}\left(\sum_{i_{2}=1}^{n_{2}}\left(\ldots\left(\sum_{i_{m}=1}^{n_{m}} w_{1_{i_{1}} 2_{i_{2}} \cdots m_{i_{m}}}\right)\right)\right) \\
\sum \frac{W^{2}}{\pi^{(m)}}=\sum_{i_{1}=1}^{n_{1}}\left(\sum_{i_{2}=1}^{n_{2}}\left(\cdots\left(\sum_{i_{m}=1}^{n_{m}} \frac{w_{1_{i_{1}} 2_{i_{2}} \cdots m_{i_{m}}}^{2}}{\pi_{1_{i_{1}}}^{\alpha} \pi_{2_{i_{2}}}^{\alpha} \cdots \pi_{m_{i_{m}}}^{\alpha}}\right)\right)\right)
\end{array}
$$

and $w_{i_{1} 2_{i_{2}} \cdots m_{i_{m}}}$ represents the total wager made on a multiple bet that pays out if all outcomes $i_{1}, i_{2}$ to $i_{m}$ within independent events $1,2,3, \ldots, m$ respectively occur.

\footnotetext{
${ }^{5}$ Refer to additional workings 1 in supplementary document.
} 
Consider multiple wagers made on the two matches shown in Figure 1. Each match has three outcomes, and therefore in total there are nine outcomes the two matches. As an example consider a total of 100 units wagered over these nine outcomes as shown Table 1 . The distribution of company profits can be calculated to follow $P^{(2)} \sim N(9.8039,20449.56)$.

Table 1: Multiple Wagers on Manchester City vs. Liverpool and Real Madrid vs. Cordoba

\begin{tabular}{|c|c|c|c|}
\hline$w_{1_{i_{1}} 2_{i_{2}} \cdots m_{i_{m}}}$ & M. City Win & Draw & Liverpool \\
\hline Real M. win & 20 & 10 & 20 \\
\hline Draw & 10 & 5 & 10 \\
\hline Cordoba win & 10 & 10 & 5 \\
\hline
\end{tabular}

PROPOSITION 6. The profit on multiples is greater than that on singles and a bookmaker is likely to make more profit if more wagers on multiples are made.

Proof (By Induction): Mathematically we need to prove that $E\left(P^{(x)}\right)>$ $E\left(P^{(y)}\right)$ where $x>y \in \mathbb{N}^{+}$.

Let $\sum W$ show the total amount of wagers made.

Let $\mathrm{m}=1$. Then: $E\left(P^{(2)}\right)=\frac{(1+k)^{2}-1}{(1+k)^{2}} \sum W=\frac{k(2+k)}{1+k} \sum W>\frac{k}{1+k} \sum_{i=1}^{n} w_{i}=$ $E\left(P^{(1)}\right)$

Let $\mathrm{m}>2$. Then $E\left(P^{(m)}\right)-E\left(P^{(m-1)}\right)=\frac{(1+k)^{m}-1}{(1+k)^{m}} \sum W-\frac{(1+k)^{m-1}-1}{(1+k)^{m-1}} \sum W=$ $\frac{k(2+k)}{1+k} \sum W>0$

$$
\therefore E\left(P^{(m)}\right)>E\left(P^{(m-1)}\right)
$$

Multiple bets are highly promoted by bookmakers. A case in point is Bwin in who promote 'Best Seller', being a bet consisting the five most popular multiples, within their main online sports betting page. The allure of multiples is for both the supply and demand end of the market: bettors are more easily enticed with higher odds (Cain, Law, \& Peel, 2003; Direr, 2013) while proposition five substantiates Zafiris' (2014) suggestion that multiples result in higher expected bookmaker profits per bet placed.

An illustration of the higher profits is a multiple bet on the outcomes of two coins (A \& B). Consider a bookmaker offering an odd of 1.8 on each outcome for each coin. Then the multiple odds would be $1.8^{2}=3.24$ on each of the four multiple outcomes ${ }^{6}$. If a total of four units are wagered on the single event of coin A (that is two units on each outcome), the expected pay-

\footnotetext{
${ }^{6}$ Both Heads, Both Tails, Coin A Head and Coin B Tails, Coin A Tail and Coin B Heads.
} 
out is $1.8 \times 2=3.6$ units. On the other hand the expected pay-out is lower at 3.24 units if four units are wagered over the multiple with two events and four multiple outcomes (that is one unit on each outcome).

Figure 2: Bestseller Option on Bwin as at 15:30 on 17th November 2014
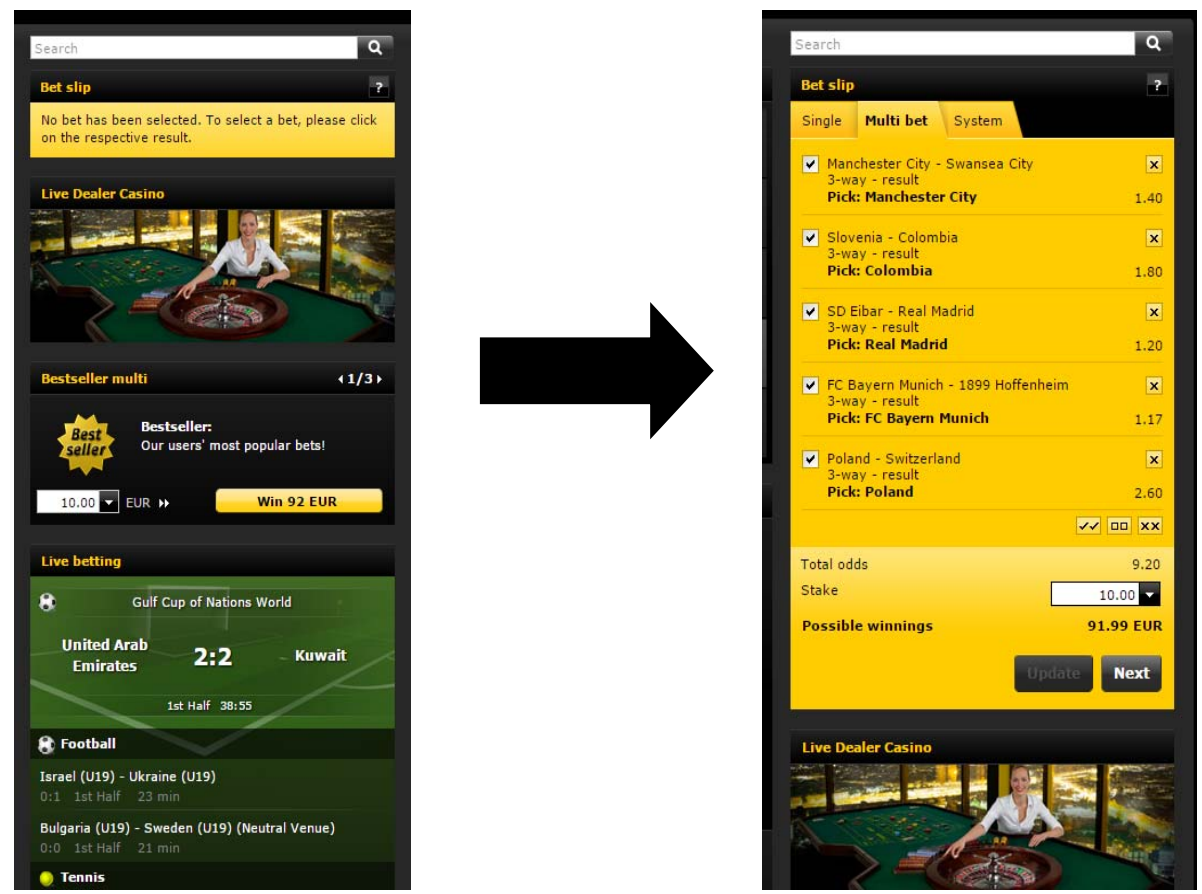

\section{CONCLUSION}

Most investigations on odds offered in betting markets have concentrated on the efficiency displayed by these odds, usually from the perspective of the bettor. This paper has provided some simple, but essential, proofs relating to the distribution of expected profit from a series of bets placed with a bookmaker.

Results point out two clear restrictions on the implied probabilities of market odds offered by bookmakers: the implied probabilities should be greater than the actual probabilities and should add up to at least $100 \%$. The commercial implications for a bookmaker are also apparent as there are larger margins of profit on multiples and profit is guaranteed if wagers are kept in ratio with implied probabilities.

\section{ACKNOWLEDGEMENTS:}

I thank Prof Jeremy Levesley (University of Leicester), Dr Frank Bezzina (University of Malta), Dr Simon Grima (University of Malta) and Dr Olga 
Lukina (University of Illinois at Chicago) for their valuable comments and recommendations. This paper is available as Open-Access thanks to a donation from Pinnacle Sports.

\section{REFERENCES}

F Archontakis and E Osborne 'Playing it safe? A Fibonacci strategy for soccer betting' (2007) 8. Journal of Sports Economics 295

S Braun and M Kvasnicka 'National sentiment and economic behavior: Evidence from online betting on European football '(2013) 14 Journal of Sports Economics 45

K H Brown and F J Abraham 'Testing market efficiency in the Major League Baseball over-under betting market' (2002) 3 Journal of Sports Economics 311

M Cain, D Law and D Peel 'The favourite-longshot bias, bookmaker margins and insider trading in a variety of betting markets' (2003) 55 Bulletin of Economic Research 263

D Cortis, S Hales and F Bezzina 'Profiting on inefficiencies in betting derivative markets: The case of UEFA Euro 2012' (2013) 7 The Journal of Gambling Business and Economics 39

B Deschamps and O Gergaud 'Efficiency in betting markets: Evidence from English football.' (2007) 1 The Journal of Prediction Markets 61

A Direr 'Are betting markets efficient? Evidence from European Football Championships' (2013) 45 Applied Economics 343

$\mathrm{S}$ Easton and K Uylangco 'An examination of in-play sports betting using one-day cricket matches.' (2007) 1 The Journal of Prediction Markets 93

J M Gandar, R A Zuber and S Johnson 'A reexamination of the efficiency of the betting market on National Hockey League Games' (2004) 5 'Journal of Sports Economics' 152

R Gil and S D Levitt 'Testing the efficiency of markets in the 2002 World Cup' (2007) 1 The Journal of Prediction Markets 255

J Goddard and I Asimakopoulos. 'Forecasting football results and the efficiency of fixed-odds betting' (2004) 23 Journal of Forecasting 51

L M Hvattum 'Analyzing information efficiency in the betting market for association football league winners.' (2013) 7 The Journal of Prediction Markets 55

A K Koch and H F Shing 'Bookmaker and pari-mutuel betting: Is a (reverse) favourite-longshot bias built in?' (2008) 2 The Journal of Prediction Markets 29.

S V Levitt 'Why are gambling markets organised so differently than betting markets?' (2004) 114 The Economic Journal 223.

R J Paul and A P Weinbach 'Market efficiency and a profitable betting rule: Evidence from totals on professional football' (2002) 3 Journal of Sports Economics 256.

D Peel and D Thomas 'The demand for football: Some evidence on outcome uncertainty' (1992) 17 Empirical Economics 323

A Schnyzter and Y Shilony 'Inside information in a betting market' (1995) 105 The Economic Journal 963

H S Shin 'Measuring the incidence of insider training in a market for state-contingent claims' (1993) 103 The Economic Journal 1141

E Štrumbelj 'On determining probability forecasts from betting odds' (2014) 30 International Journal of Forecasting 934 
D Terrell and A Farmer 'Optimal betting and efficiency in parimutuel betting markets with information costs' (1996) 106 The Economic Journal 846

L Vaughan Williams and D Paton 'Why is there a favourite-longshot bias in British racetrack betting markets?' (1997) 107 The Economic Journal 150

L M Woodland and B M Woodland 'Market efficiency and profitable wagering in the National Hockey League: Can bettors score on longshots?' (2001) 67 Southern Economic Journal 983

N Zafiris 'When is a multiple bet better than a single?' (2014) 8 The Journal of Gambling Business and Economics 1 


\section{APPENDIX A - PAYOUT DISTRIBUTION FOR CONSTANT $\mathbf{k}$}

Proposition: If $\pi_{i}=p_{i}(1+k)$ for some constant $\mathrm{k}$; then $P \sim N\left(\frac{k}{1+k} \sum_{i=1}^{n} w_{i}, \frac{1}{(1+k)^{2}}\left(\sum_{i=1}^{n} \frac{w_{i}^{2}}{p_{i}}-\left(\sum_{i=1}^{n} w_{i}\right)^{2}\right)\right)$

Proof: $E(P)=E($ Wagers less Payouts $)=\sum_{i=1}^{n}\left(p_{i} \sum_{i=1}^{n} w_{i}-w_{i} \times \frac{1}{\pi_{i}} \times p_{i}\right)$ $E(P)=\sum_{i=1}^{n} w_{i}-\sum_{i=1}^{n} \frac{w_{i}}{1+k}=\frac{k}{1+k} \sum_{i=1}^{n} w_{i}$

$E\left(P^{2}\right)=E\left((\text { Wagers less Payouts })^{2}\right)=\sum_{i=1}^{n}\left(\sum_{i=1}^{n} w_{i}-w_{i} \times \frac{1}{\pi_{i}}\right)^{2} \times p_{i}$

$E\left(P^{2}\right)=\sum_{i=1}^{n}\left[\left(\sum_{i=1}^{n} w_{i}\right)^{2} p_{i}-\frac{2 w_{i}}{1+k} \sum_{i=1}^{n} w_{i}+\frac{1}{p_{i}}\left(\frac{w_{i}}{1+k}\right)^{2}\right]$

$E\left(P^{2}\right)=\left(1-\frac{2}{1+k}\right)\left(\sum_{i=1}^{n} w_{i}\right)^{2}+\frac{1}{(1+k)^{2}} \sum_{i=1}^{n} \frac{w_{i}^{2}}{p_{i}}$

$E\left(P^{2}\right)=\frac{k-1}{1+k}\left(\sum_{i=1}^{n} w_{i}\right)^{2}+\frac{1}{(1+k)^{2}} \sum_{i=1}^{n} \frac{w_{i}^{2}}{p_{i}}$

$\operatorname{Var}(P)=E\left(P^{2}\right)-[E(P)]^{2}$

$$
=\frac{k-1}{1+k}\left(\sum_{i=1}^{n} w_{i}\right)^{2}+\frac{1}{(1+k)^{2}} \sum_{i=1}^{n} \frac{w_{i}^{2}}{p_{i}}-\frac{k^{2}}{(1+k)^{2}}\left(\sum_{i=1}^{n} w_{i}\right)^{2}
$$

$\operatorname{Var}(P)=\frac{1}{(1+k)^{2}}\left(\sum_{i=1}^{n} \frac{w_{i}^{2}}{p_{i}}-\left(\sum_{i=1}^{n} w_{i}\right)^{2}\right)$ 